\title{
tic\&société
}

Vol. 10, $N^{\circ} 1 \mid 1$ er semestre 2016

Contrôle social, surveillance et dispositifs numériques

\section{Marshall McLuhan et la théorie médiatique : genèse, pertinence et limites d'une contribution contestée}

\section{Oumar KANE}

\section{(2) OpenEdition Journals}

Electronic version

URL: https://journals.openedition.org/ticetsociete/2043

DOI: 10.4000/ticetsociete.2043

\section{Publisher}

Association ARTIC

\section{Electronic reference}

Oumar KANE, "Marshall McLuhan et la théorie médiatique : genèse, pertinence et limites d'une contribution contestée", tic\&société [Online], Vol. 10, № 1 | 1 er semestre 2016, Online since 06 November 2016, connection on 21 September 2021. URL: http://journals.openedition.org/ticetsociete/ 2043 ; DOI: https://doi.org/10.4000/ticetsociete.2043

This text was automatically generated on 21 September 2021.

Licence Creative Commons 


\title{
Marshall McLuhan et la théorie médiatique : genèse, pertinence et limites d'une contribution contestée
}

\author{
Oumar KANE
}

\section{Introduction}

1 Aucune bibliographie en sciences de la communication, aussi minimaliste soit-elle, ne pourrait faire l'économie des travaux de M. McLuhan. Cela même si le théoricien canadien des media ${ }^{1}$ devait se retrouver dans le peloton de tête des auteurs les plus critiqués de la discipline. Sa grande médiatisation, son style d'écriture (très éloigné des canons de la rigueur scientifique) et les controverses dans lesquelles il s'est engagé ne sont certainement pas étrangers à cet état de fait. Formé aux humanités classiques, il ne s'interdira cependant pas de prendre pour objet d'étude la culture populaire et les " nouveaux médias », au rang desquels figurait au début des années 1960 la télévision.

L'intérêt de McLuhan pour les media et la culture prend véritablement son essor avec l'obtention d'une subvention octroyée par la Fondation Ford au début des années 1950. Le théoricien canadien, qui s'intéressait auparavant, dans le prolongement de sa thèse de doctorat, principalement à la littérature et à la critique anglaises (McNamara, 1969 ; Ong, 1970 ; Stamps, 1995), élargira ensuite son domaine de recherche à d'autres objets et à d'autres publics (Buxton, 2012).

Mais le propos de McLuhan dépasse de loin le cadre du strict confinement à la dernière technologie à la mode puisqu'il développe une conception extrêmement vaste du medium qu'il articule avec l'appareil perceptif humain dans le cadre d'une expérience sensorielle, c'est-à-dire une esthétique. Si son projet d'analyser les media et d'en dresser une typologie est salué par ses pairs et reconnu aujourd'hui comme une initiative pionnière et significative en théorie médiatique - McLuhan figure au panthéon des auteurs adulés par l'écologie médiatique contemporaine -, il s'est par ailleurs exposé à des critiques acerbes 
au point d'être associé de manière quasi systématique au déterminisme technologique. D'où l'interrogation, maintenant classique, relative à sa réception (Jeffrey, 1989, p. 1) :

\begin{abstract}
«Continuera-t-on à le considérer ainsi qu'on l'entend souvent comme un personnage controversé, un homme marginal venant d'un pays marginal et qui s'est fourvoyé? Sera-t-il considéré comme une icône des années 1960 qui a contribué de manière importante à changer la manière dont la plupart des gens se représentent les media? Ou son importance sera-t-elle évaluée à l'aune du rôle qu'il a joué en tant que catalyseur dans le développement de la communication comme "interdiscipline"? ${ }^{2}$
\end{abstract}

Dans le cadre du présent article, notre objectif n'est pas de répéter les nombreuses évaluations qui ont été faites des travaux de McLuhan, mais plutôt d'évoquer succinctement certains d'entre eux et, avec le recul temporel dont nous disposons aujourd'hui, de considérer la postérité de ses idées dans les études en communication actuelles.

Pour ce faire, nous tenterons de montrer que, malgré leurs limites, les travaux de McLuhan ont encore une certaine pertinence pour l'analyse des phénomènes médiatiques et que sa contribution à la théorie médiatique mérite d'être évaluée de manière plus équilibrée que le partage tranché entre ses détracteurs et ses épigones ne le laisse trop souvent paraître. En premier lieu, nous retracerons la genèse de son œuvre et nous mettrons au jour ses propres dettes envers certains de ses maitres à penser. Son approche esthétique des media y sera mise en relation avec certaines traditions de recherche importantes en études littéraires, de manière à mieux contextualiser sa contribution théorique. Ensuite, nous évoquerons la réception de ses travaux et nous rendrons compte tant des critiques les plus récurrentes qui lui ont été adressées que de sa postérité, c'està-dire les dettes que la communauté des chercheurs en communication a collectivement contractées à son égard, que ce soit de manière explicite ou implicite. Avant de conclure, nous terminerons notre développement par l'évocation de ses relations avec la communication non plus comme phénomène, mais comme (inter)discipline ou quasidiscipline (Kane, 2010). McLuhan a en effet joué un rôle important dans l'institutionnalisation de la communication et est entré en dialogue avec des auteurs importants d'un champ alors en voie de constitution.

\title{
1. Genèse et étendue des travaux de McLuhan
}

\subsection{Tradition littéraire contre rigueur scientifique}

6 Un siècle après sa naissance et une trentaine d'années après son décès, M. McLuhan (1911-1980) est l'objet de nombreuses célébrations et de relectures critiques ou dithyrambiques visant à évaluer la portée de sa contribution à divers champs disciplinaires, particulièrement en sciences de la communication. Paradoxalement, il semblerait que si l'histoire des communications est devenue un champ de recherche à part entière, c'est à la fois grâce et en dépit des travaux de McLuhan (Heyer, 1989) ; ce qui est révélateur de la réception dont ont bénéficié les travaux de l'auteur canadien. Adulé pendant les années 1960, délaissé vers la fin de sa vie, McLuhan est revenu en force dans les années 1990. L'engouement pour le poststructuralisme semble avoir joué un rôle important dans ce regain d'intérêt. En déconstruisant et en problématisant l'objectivité, le savoir scientifique et la rationalité, le poststructuralisme aurait ainsi désamorcé les 
arguments des détracteurs de McLuhan, permettant à ses idées d'acquérir une dignité nouvelle dans le champ scientifique et d'effectuer, à titre posthume, un retour en force (Wilmott, 1996). Au début des années 1960, sa notoriété mondaine et médiatique connaît un développement important, au point de précéder sa réception scientifique et de la biaiser notablement selon certains auteurs. Caricaturé et réduit à deux aphorismes simplistes ${ }^{3}$ - «le medium est le message » et le «village global»-, servi par un sens aigu de la formule, mais desservi par un style aphoristique et parfois jugé hermétique, McLuhan jouit d'une postérité tout sauf facile à évaluer tant les prises de position sont tranchées, malgré certaines tentatives de nuancer le propos (Rosenthal, 1969).

McLuhan a préconisé une approche pionnière et inédite de l'analyse des media, à un moment où la recherche en communication se déployait dans des directions radicalement différentes. Sa première monographie, The Mechanical Bride: Folklore of Industrial Man (1951), constitue à plusieurs égards une anomalie (sur laquelle nous reviendrons) dans l'ensemble de son œuvre. The Gutenberg Galaxy: The Making of the Typographic Man (1962) analyse les transformations culturelles consécutives à l'invention de l'imprimerie. L'ouvrage sera suivi, en 1964, par le désormais classique Understanding Media: The Extensions of Man. Les sous-titres de ses ouvrages sont souvent très éloquents et permettent de saisir véritablement la thèse qui y sera développée. Dans The Medium is the Massage 4 : An Inventory of Effects paru en 1967, en collaboration avec Q. Fiore et J. Agel, il écrit que le fait que les media électroniques sont non plus l'extension d'un sens unique (comme le livre, extension de l'œil, ou le langage, celle de l'oreille) mais du système nerveux humain dans son ensemble a des conséquences très importantes. L'abolition de la conception linéaire de l'espace et du temps est un caractère essentiel selon McLuhan, puisqu'elle marque le passage à l'époque du post-imprimé. C'est paradoxalement à travers un livre imprimé richement illustré par Q. Fiore que McLuhan a annoncé la mort du livre (Macey, 2001).

8 Le succès de McLuhan auprès du grand public serait dû, selon Winter et Goldman (1989, p. 99), à sa «théologie séculière de la technologie électronique ». Ces deux auteurs publient leur article en 1989, dans une conjoncture caractérisée par une vague d'évaluation des travaux de McLuhan en vue de déterminer l'importance de son œuvre pour une sociologie de la connaissance. Les termes utilisés pour rendre compte de ses travaux oscillaient entre la simple « réputation » mondaine et la « fondation » des media studies, en passant par diverses formes de reconnaissance d'une contribution à l'histoire des idées ou à la production de connaissances.

\subsection{Les dettes de McLuhan}

9 Si l'on en croit Babe (2000), quatre auteurs ont exercé une influence majeure sur McLuhan. Il s'agit d'abord de L. Mumford, auteur de Technics and Civilization en 1934, qui a analysé les relations entre la technologie et la culture. S. Giedion, ensuite, qui a écrit Space, Time and Architecture en 1941 et Mechanization Takes Command en 1948, l'a influencé à travers ses thèses sur la mécanisation de la conscience. E. Havelock, membre de l'école de Toronto, aurait grandement impressionné McLuhan par ses études sur le déséquilibre civilisationnel survenu suite à l'invention de l'alphabet phonétique chez les Anciens. Havelock développe notamment ses thèses dans The Crucifixion of Intellectual Man en 1950 et dans Preface to Plato en 1963. Enfin, last but not least, H. Innis, dont l'influence sur McLuhan est la plus largement attestée à travers son insistance sur l'importance des 
media dans l'exercice du pouvoir et dans le changement sociétal, qu'il a notamment traité dans Empire and Communications en 1950 et dans The Bias of Communication en 1951.

McLuhan doit en partie l'élargissement radical de la définition du medium qu'il opère à Giedion, qui considérait que tout objet peut être "esthétique ", en d'autres termes, être étudié sous l'angle de ses rapports avec l'appareil perceptif humain. Prenant au sérieux cet enseignement, McLuhan considère comme « medium » tout objet, artefact ou dispositif qui entretient une relation avec le sensorium humain, tous les artefacts techniques qui prolongent des fonctions, des facultés ou des organes sensoriels humains. Aussi le vêtement, la route ou la roue sont-ils des media qui ont des "effets " psychologiques et sociétaux très importants. Par ce biais, l'analyse des media a eu pour effet inattendu de replacer le corps au centre de l'analyse chez certains auteurs qui se revendiquent de McLuhan, considérant ainsi que « l'ultime leçon de Marshall est que le corps est le média, et qu'il est notre principal atout » (Coupland, 2009, p. 232).

11 La dette de McLuhan envers le new criticism ${ }^{5}$ mérite également d'être mentionnée car elle permet de comprendre avec beaucoup plus de clarté sa démarche sur les plans de la forme et du contenu. Le new criticism, qui émerge dans les années 1930 dans le champ de la critique littéraire britannique et américaine, subit notamment son influence par l'entremise de deux de ses professeurs à Cambridge, I. A. Richards et F. R. Leavis, qui étudiaient la manière dont les aspects formels de la littérature influencent la réception des lecteurs. Sans avoir la cohésion d'une " école », ses tenants élaborent et partagent un discours et une pratique ancrés dans la poésie et qui s'opposaient à la critique philologique et historique. Cette dernière analysait les œuvres en portant un intérêt quasi exclusif au contenu du texte par le biais d'une herméneutique textuelle appliquée à la poésie. Dès lors, « la tâche du critique est par conséquent de procéder à "l'analyse la plus minutieuse possible de ce que le poème signifie en tant que poème"6 " (Macey, 2001, p. 269). On voit assez bien ici la filiation avec le désintérêt qu'éprouvait McLuhan pour le contenu des media qui ont à être analysés « en tant que media ». Le new criticism s'insurge contre deux biais majeurs de la critique littéraire, qui consistent à centrer l'analyse sur l'intention (intentional fallacy) et l'affectivité (affective fallacy). Il s'agit de biais parce que, selon les nouveaux critiques, l'intention de l'auteur d'une œuvre n'est pas disponible pour l'analyste et il ne servirait à rien de se perdre en conjectures à son propos. De même, insister sur l'affectivité reviendrait à perdre de vue le "texte en tant que texte». Le paradoxe et la métaphore s'y voient accorder une importance nouvelle. Le résultat est une prise de distance du discours scientifique et la revendication d'un discours non scientifique, paradoxal et aphoristique (Macey, 2001), qui aura les faveurs de McLuhan et qui déroutera grandement plusieurs de ses lecteurs.

L'influence du new criticism apparait dans la manière dont McLuhan appliquera l'analyse des formes textuelles à celle des formes technologiques que sont à ses yeux les nouveaux media. C'est notamment en conjuguant les travaux d'Innis avec les thèses du new criticism que la conception mcluhanienne selon laquelle les media perçus comme des formes engendrent des effets analysables en tant que biais sur le sensorium s'éclaire sous un jour nouveau: "Les effets des nouveaux media sur notre vie sensorielle sont similaires aux effets de la nouvelle poésie. Ils changent non pas nos pensées, mais la structure de notre monde ${ }^{7}$ (McLuhan, cité par Marchessault, 2005, p. 16). 


\subsection{Une approche esthétique des media}

13 S'il convient de tracer une ligne de partage entre un premier et un deuxième McLuhan dans son approche ${ }^{8}$ des media, The Mechanical Bride (1951) appartiendrait sans aucun doute à la première période, pendant laquelle le théoricien a déployé une critique acerbe de la culture populaire et cloué au pilori les milieux du business et de la publicité. Cette dénonciation de la consommation de masse et de la publicité disparaitra de ses travaux ultérieurs, notamment ceux du début des années 1960 (The Gutenberg Galaxy et Understanding Media). McLuhan reniera plus tard The Mechanical Bride en le qualifiant de tentative insensée de se raccrocher à un passé révolu dans une société alors dominée par les nouveaux médias ${ }^{9}$ qu'étaient à l'époque la radio et la télévision. C'est ce qui fait dire à Winter et Goldman $(1989$, p. 96) : «C'est quelque part dans cette période de onze ans qui s'étend de 1951 à 1962 que Marshall McLuhan est devenu un déterministe technologique ${ }^{10}$ ». Avec ce déplacement analytique, le locus de responsabilité (au sens non moral du terme) est désormais technologisé et extériorisé ; il est à chercher dans les media eux-mêmes au lieu d'être mis au débit du système et des institutions de communication comme auparavant. On entre ainsi dans un univers déterministe dans lequel les media sont tantôt à chérir tantôt à blâmer. Cependant, la portée critique de l'analyse médiatique de McLuhan subsiste virtuellement avec la possibilité que, poussée à l'extrême, la logique de fonctionnement d'un medium s'inverse pour actualiser des potentialités contraires à son mode de fonctionnement dominant. C'est le chiasme ${ }^{11}$ auquel réfère Babe $(2010$, p. 299) dans son analyse de la pensée communicationnelle de McLuhan.

La thèse de McLuhan, soutenue à l'université de Cambridge en 1943, portait sur Thomas Nashe et était intitulée The Classical Trivium ${ }^{12}$ : The Place of Thomas Nashe in the Learning of His Time. Il s'y intéressait à l'histoire de la rhétorique dans la période allant de l'Antiquité à la Renaissance. De là naquit son intérêt pour les technologies de communication et leur relation/influence avec l'histoire sociale des peuples. En concevant le langage comme une technologie, McLuhan a pu considérer sur le même continuum les artefacts sous l'angle de leurs effets sur le sensorium ${ }^{13}$ humain, défini par saint Thomas d'Aquin comme l'interaction des cinq sens. Il est parvenu par ce biais à explicitement faire équivaloir artefact et medium. Ce faisant, il a élargi le domaine initial d'application (le langage) de la critique littéraire non seulement aux media au sens restreint du terme, mais à tout medium conçu au sens large préalablement mentionné (la roue ou le vêtement, par exemple). Comme il l'affirme dans Counterblast, il a ainsi essayé de développer une « approche écologique » qui prend conjointement en compte les media et les sens humains qu'ils prolongent ou accentuent. Mais alors que les media mécaniques amplifiaient un sens au détriment des autres, l'avènement de l'ère électronique opère un changement radical en extériorisant le système sensoriel humain dans son ensemble. Il s'agit là d'un changement majeur dans l'histoire des communications et dans l'écologie médiatique.

Dans la même perspective, une autre innovation mcluhanienne relative à l'analyse des media, très controversée, concerne la «température » de ces derniers. McLuhan classe en effet les media en deux catégories, chauds et froids (Heyer, 1989, p. 41) :

« Un medium chaud est celui qui amène un sens unique à un niveau de haute définition ; il est riche en informations spécifiques et laisse peu de place à l'activité des individus. Selon McLuhan, l'imprimerie est chaude, tout comme la radio et le cinéma. À l'opposé, les media froids sont à basse définition en termes d'information. Ils nécessitent la participation du public, ce qui 
implique la sollicitation de plusieurs sens. L'oralité est froide, de même que le téléphone et la télévision $»^{14}$. lêtre humain change en présence des technologies. Par ailleurs, toute époque connaît un medium d'élection dont le mode relationnel aux sens sera dominant pendant la durée de son hégémonie. Par conséquent, toute transformation technologique des media transforme l'environnement social et, par ricochet, la perception individuelle et collective. C'est pourquoi McLuhan parle parfois des media en termes d'environnement. Dès lors, la matérialité du medium précède et prime celle du contenu contingent qu'il peut lui arriver de véhiculer. Cela ne suffit pas à remettre en cause le primat du medium « en tant que medium ». La distinction entre contenu et message aboutit à l'assertion selon laquelle le contenu de la télévision est constitué par les images tandis que son message est lié à ses effets sur les téléspectateurs.

Là encore, certaines prises de position du McLuhan spécialiste de la rhétorique permettent de comprendre le McLuhan théoricien des media. En effet, il s'est régulièrement insurgé contre les approches nominalistes et a défendu l'idée selon laquelle les mots ne sont pas arbitraires car ils tirent leurs racines de l'expérience. Il part de l'analyse des onomatopées et de la poésie (le mot est lié esthétiquement à la réalité qu'il désigne) pour l'appliquer aux media qui, dans son approche, maintiendraient de ce fait une relation motivée aux sens humains.

Il importe de préciser que, pour McLuhan, il est vrai que les media comme extensions ne s'appliquent qu'à l'espèce humaine, mais ils s'appliquent universellement ${ }^{15}$ à l'espèce humaine. Dès lors, il a cherché à trouver une réponse à la question : «Que font tous les médias? » Son ambition était de définir des lois à portée générale et qui sont vérifiables. Dans un article qui tente de faire le point sur la contribution scientifique de son père, $E$. McLuhan (1989, pp. 80-81) estime que M. McLuhan a identifié quatre lois qu'il a formulées sous forme de questions en vue de donner une réponse à son questionnement global : «Que font tous les media?»

Ces questions, qui sont à poser à propos de chaque medium dominant dans une conjoncture historique donnée, sont les suivantes : 
basculer soudainement ${ }^{16}$ " (McLuhan et Nevitt, 1972, p. 6). Les questionnements susmentionnés permettent de saisir la virtualité de ce basculement potentiel. Est ici mobilisée une analyse, lancée par Understanding Media et complétée par From Cliché to Archetype (1970) qui évite à McLuhan, au moins en théorie, de tomber dans une analyse exagérément déterministe. Cette formalisation permet au contraire de comprendre comment les tendances que les media favorisent en arrivent à s'inverser quand leur logique est poussée à l'extrême. Ainsi, l'approche mcluhanienne des media est certes déterministe, mais elle n'est pas mécaniquement univoque puisqu'elle est susceptible de s'inverser pour ce qui est de ses effets.

\section{La réception de McLuhan}

\subsection{McLuhan critiqué}

Les critiques adressées à McLuhan sont légion et il serait impossible de tenter de les aborder exhaustivement ici. Nous en évoquerons très brièvement quelques-unes en les contextualisant dans le cadre des jeux d'acteurs dans lesquels elles ont émergé. Cela nous servira de contrepoint quand nous évoquerons sa postérité dans la section suivante.

Issu de la critique littérature anglaise et partisan du new criticism, McLuhan est, au début des années 1960, un outsider dans le champ de la communication. Il se place en porte-àfaux vis-à-vis des paradigmes dominants d'un champ en voie d'institutionnalisation. La situation du new criticism par rapport à la critique philologique est en effet homologue de celle de l'analyse mcluhanienne des media par rapport aux approches sociologiques dominantes (effets psychologiques immédiats, analyse de la propagande ou psychosociologie de la communication de masse). En décidant de mettre l'accent sur les media " en tant que media ", il s'expose à la critique faite au new criticism dans son propre espace de déploiement. C'est-à-dire qu'en analysant le "poème comme poème ", la critique littéraire et le poème se referment sur eux-mêmes, aboutissant à un système autoréférentiel qui ne peut donc que s'autoconfirmer tautologiquement. La même critique a été faite à McLuhan dans son approche des media, et ce n'est en rien un hasard, en raison de l'homologie structurelle (Bourdieu, 1980) dans laquelle le new criticism et sa théorie des media se trouvent par rapport respectivement à la critique littéraire britannique mainstream et aux communication studies américaines.

La critique la plus récurrente adressée à McLuhan est probablement celle lui reprochant de promouvoir une approche déterministe des media : «Par déterminisme technologique, on entend l'attitude intellectuelle qui consiste à croire que la technologie détermine essentiellement ou premièrement l'organisation sociale et le comportement humain " (Attallah, 1989, p. 290). La critique du technodéterminisme pointe la posture mcluhanienne qui énonce le primat des media $^{17}$ sur tous les autres facteurs dans le développement historique. Une autre critique, qui va plus loin, s'attaque à la monocausalité de l'auteur canadien : « Le problème, naturellement, est que la causalité, si elle existe dans l'histoire, ne peut pas être attribuée à un facteur unique comme les media, en particulier dans le cas des longues périodes historiques traitées dans La Galaxie Gutenberg $^{18} »\left(\right.$ Heyer $^{19}, 1989$, p. 37). Heyer reproche à McLuhan de réduire le changement social à la transformation induite exclusivement par les media au mépris des autres déterminants sociohistoriques. 
L'appropriation par McLuhan des travaux d'Innis est également un sujet controversé (Marchand, 1990). Buxton (2012), par exemple, soutient que McLuhan a effectué une lecture partielle et orientée de l'œuvre d'Innis en vue de se construire une crédibilité dans le champ des media et de la culture. Il aurait ainsi réinterprété de manière spécifique les écrits d'Innis, notamment à travers sa préface de la réédition de The Bias of Communication (1964) dans laquelle il attribue à Innis une définition réductrice des media contre laquelle s'insurge Buxton (2012, p. 583) :

«[...] Le concept innissien de communication ne pouvait pas être réduit à une simple forme dérivée des media, il devait plutôt être davantage considéré comme un processus interactif, intrinsèquement lié au progrès civilisationnel, à la naissance des universités et à l'avènement de nouvelles formes de public... Les media, dans l'acception d'Innis, vont bien au-delà des "ressources économiques", auxquelles McLuhan a réduit les travaux d'Innis consacrés à l'histoire des communications $»^{20}$.

À l'opposé, Jeffrey (1989), dans ce qui s'apparente à une entreprise de défense de McLuhan, soutient que Carey ${ }^{21}$ (1970) a plus que tout autre chercheur contribué à ternir sa réputation dans le champ des sciences sociales quand il opère une analogie entre les thèses mcluhaniennes et l'hypothèse Sapir-Whorf ${ }^{22}$. La charge de Carey contre la célèbre hypothèse aurait servi à décrédibiliser indirectement les travaux du théoricien canadien des media. Ce faisant, Carey aurait choisi de magnifier l'œuvre d'Innis, plus proche de son orientation idéologique, au détriment des travaux de McLuhan à cause de l'absence de perspective critique dans la quasi-totalité des travaux de ce dernier (à l'exception notable de The Mechanical Bride).

Malgré l'existence d'une vaste littérature critique de McLuhan, dont certains auteurs ont été ses étudiants, ses idées ont connu une seconde vie et ont été largement reprises, tantôt par de simples épigones, tantôt dans le cadre d'appropriations plus fructueuses. Nous allons évoquer dans la section suivante quelques approches qui se revendiquent aujourd'hui explicitement de l'approche mcluhanienne des médias.

\subsection{La postérité de McLuhan}

La contribution de McLuhan à la recherche dans le domaine de la communication est manifeste et se mesure aisément par le succès que ses thèses ont reçu et par les citations dont il fait l'objet dans les recherches actuelles sur les media et la communication. L'influence du théoricien canadien a largement débordé le cadre de la discipline communicationnelle puisque des auteurs aussi divers que Z. Brzezinski (1970), D. Bell (1973) ou A. Toffler (1980) se revendiquent plus ou moins ouvertement de son héritage. Aussi, même Heyer, dont nous venons d'évoquer la force de la critique adressée aux écrits de McLuhan, reconnaît-il la réalité de son apport à la théorie contemporaine des médias (Heyer, 1989, p. 44) :

« Néanmoins, aucune discussion contemporaine sur rôle de la télévision, y compris celle basée, "à Dieu ne plaise", sur le contenu, ne peut faire l'économie de la perspective de McLuhan. Même l'évitement complet de ses travaux entraine une situation où des considérations importantes surgissent inévitablement entre le point de vue non McLuhanien et celui qu'il tente de contourner ou de contredire. Malgré ses lacunes théoriques, Understanding Media est un livre important. Son importance ne réside pas dans ce qu'il dit à 
propos de choses, mais plutôt dans le réseau de relations qu'il établit entre les choses qu'il analyse $»^{23}$. des media. En France, la médiologie de R. Debray, qui étudie les effets des appareillages et des institutions sur la pensée individuelle et collective, lui doit beaucoup dans la mesure où les médiologues s'intéressent aux régimes symboliques (de savoir, de mémoire, d'institution, etc.) en rapport avec les outils et les artefacts. La médiologie française insiste sur les media comme "matière organisée » (et par là elle emprunte à McLuhan), mais elle prend également en compte les organisations dans leur sens large pour analyser ce qui relève des « transmissions ». L'apport mcluhanien est donc approprié dans le cadre du projet médiologique et articulé à une prise en compte des institutions. Il demeure que la conception de sphères successives (logosphère, graphosphère, vidéosphère) n'est pas sans rappeler l'étapisme historique développé par l'auteur canadien (galaxie Gutenberg, galaxie Marconi, village global). À cet égard, la médiologie en tant que «science du milieu » dans laquelle les media ont un rôle prépondérant s'inscrit clairement dans la postérité mcluhanienne. L'élargissement médiologique de l'univers des media à tous les artefacts techniques ne fait pas non plus mystère de sa dette envers le théoricien canadien :
"L'enquête sur les effets culturels des médiations techniques ne peut en effet ignorer des objets comme la bicyclette, l'horloge, la route ou le télescope. Autant de media qui ne communiquent aucun message explicite, mais qui accordent les pianos de nos relations, en réglant nos rapports à l'espace et au temps » (Marzeau, 1998, p. 31).

La référence à la conception mcluhanienne des media ne saurait être plus manifeste.

L'écologie des media est également héritière de McLuhan. L'auteur canadien est abondamment cité dans les travaux qui s'en revendiquent, même s'il cohabite au panthéon de l'écologie des media avec plusieurs autres noms illustres comme L. Mumford, E. Havelock ou W. Ong. Née du vivant de McLuhan, dans les années 1970, l'écologie des media cherche à mieux cerner les rapports entre la société, la culture et la technologie. Développant une approche systémique, elle s'efforce de penser le medium à travers sa matérialité pour saisir les rapports qui se nouent, se construisent et se défont avec le temps (Meyrowitz, 1985). Ainsi compris, le projet ne pouvait faire l'économie des travaux de McLuhan qui ont notamment influencé N. Postman (1993), l'un des auteurs les plus importants de l'écologie des media et qui lui a redonné un souffle nouveau dans les années 1990 et 2000.

En sémiotique des media également, le legs de McLuhan se fait plus manifeste ces dernières années. En s'interrogeant sur le statut du support médiatique, la recherche dans le domaine ne peut faire l'impasse sur les travaux de l'auteur canadien. L'intérêt pour la matérialité des supports et les effets technognosiques, c'est-à-dire conjointement physiques et cognitifs, trouve chez McLuhan les outils conceptuels pour penser le medium comme support (d'inscription, de mémoire ou de prolongement des sens), comme organon, en vue de construire un terrain de rencontre entre la technique, la société et l'humain.

37 Nous allons aborder dans la dernière partie la communication en tant que discipline et champ de recherche en évoquant quelques enjeux dont McLuhan a été de son vivant l'un des acteurs principaux. 


\section{Pour une conception de la communication comme interdiscipline?}

38 L'école de Toronto, dont E. Havelock, H. Innis et M. McLuhan figurent parmi les membres les plus illustres, s'est attelée à penser les effets des technologies de communication (dont l'écriture) sur le fonctionnement des sociétés humaines à travers l'espace et le temps. Elle a été déterminante dans la fondation de l'histoire des communications comme champ de recherche autonome (Buxton, 2012). À cet égard, alors qu'Innis travaillait clairement à partir d'un ancrage disciplinaire en histoire économique, McLuhan aura contribué de manière importante à l'institutionnalisation des études en communication en favorisant résolument l'interdisciplinarité (Jeffrey, 1989, p. 16) :

«Institutionnellement, McLuhan a contribué au développement de l'interdisciplinarité de plusieurs manières. Son approche interdisciplinaire était manifeste dans la diversité des disciplines de provenance des chercheurs invités à participer aux Séminaires sur les communications et la culture tenus de de 1953 à 1955 à l'Université de Toronto et financés par la Fondation Ford... La subvention de la Fondation Ford en 1953 a permis de stimuler la collaboration et les recherches conjointes dans le cadre de l'École de communication de Toronto $»^{24}$.

39 Ce n'est d'ailleurs pas un hasard si la volonté de concevoir la communication comme interdiscipline est apparue chez des universitaires venant de champs disciplinaires différents et rassemblés principalement autour de l'étude de l'histoire et des phénomènes de communication. Le fait que le doctorat en communication de l'université McGill à Montréal ait été fondé par D. Theall, l'un de ses anciens étudiants à l'université de Toronto, a poussé McLuhan à tenter de promouvoir un programme en communication à Toronto et à solliciter pour ce faire l'aide d'Innis. Quelle que soit la fortune que ce projet a connue, McLuhan est sans conteste l'un des auteurs qui ont le plus contribué à fédérer des chercheurs autour des médias et de la communication au Canada (Jeffrey, 1989). À partir de 1953, la revue Explorations: Studies in Culture and Communication devient un terreau fertile pour des universitaires issus de disciplines diverses, mais animés par un intérêt commun pour l'étude des phénomènes de communication. De sorte que certaines des thématiques développées en 1962 dans The Gutenberg Galaxy sont tirées d'idées déjà développées par McLuhan et d'autres auteurs dans Explorations.

Ce n'est cependant pas pour autant qu'un consensus existait parmi les chercheurs nordaméricains puisqu'un conflit a opposé McLuhan et certains théoriciens majeurs de la communication aux États-Unis. Le théoricien canadien a notamment critiqué W. Schramm et P. Lazarsfeld dans Understanding Media leur reprochant d'être les victimes d'une ignorance épistémique dans leur analyse de la télévision (Schramm) et de la radio (Lazarsfeld). Il considère être pour sa part un innovateur dans la recherche sur les médias (cité dans Jeffrey, 1989, p. 16) :

« La raison pour laquelle je suis admiré à Paris et dans certains pays latins est que mon approche est considérée à juste titre comme "structuraliste". Je l'ai acquise à travers Joyce et les symbolistes puis utilisée dans The Mechanical Bride. Personne, dans le domaine des médias, hormis moi-même, ne s'est risqué dans l'approche structuraliste ou "existentielle". C'est une approche 
savante, or les écoles de communication sont uniformément technocentrées et frileuses dans leur formation et leurs activités ${ }^{25}$.

41 Dans les années 1960, quand McLuhan s'intéresse véritablement aux techniques et supports de communication, la recherche sur les media et la communication de masse est dominée par des approches linéaires, béhavioristes ou quantitatives héritées des psychologues et des sociologues qui œuvrent dans le champ. La perspective inédite de McLuhan, qui se démarque fortement des traditions en voie d'institutionnalisation, lui fait affirmer que la force de son approche réside dans l'approche de transformation qu'il offre, tandis que les ténors du champ analysent encore la communication sous le registre du transport.

Le fait que McLuhan était un outsider issu de la littérature anglaise lui a permis de renouveler l'analyse des media à l'instar de ce qui s'est passé en Grande-Bretagne avec des auteurs provenant également de la théorie littéraire anglaise, comme R. Williams, qui ont renouvelé de manière similaire l'analyse médiatique (Jeffrey, 1989). Mais alors que Williams s'inscrivait dans une perspective critique, McLuhan a subi les foudres des théoriciens critiques parce qu'il laissait de côté les relations de pouvoir que seules l'analyse du contenu et celle des conditions de production permettaient de mettre au jour.

\section{Conclusion}

Les théories innissienne et mcluhanienne sont des théories communicationnelles culturalistes ${ }^{26}$ qui s'attachent à faire une histoire sociale de la communication. La différence, essentielle, réside cependant en ce qu'Innis attribue des " qualités historiques aux media " tandis que McLuhan attribue des "qualités médiatiques à l'histoire " (Attallah, 1989, p. 275). Par le prolongement des facultés ou des organes humains, les media parviennent par leur simple matérialité à induire un changement d'échelle des activités humaines. L'électricitén ${ }^{27}$, medium des media, parviendrait à affecter l'ensemble des autres media alors que ces derniers affectent pour leur part le sensorium humain. L'électricité serait de ce fait proche d'un meta medium à l'intérieur de ce qu'on pourrait appeler l'écologie médiatique mcluhanienne.

Malgré cette détermination en première instance de l'échelle des activités humaines par les media, qui a fait l'objet de très nombreuses critiques (comme beaucoup d'autres aspects des écrits de McLuhan), force est de reconnaître que l'analyse de la retribalisation qu'il fait dans le cadre de la société moderne montre que les effets des media ne sont pas unilinéaires et que des hybridations d'effets peuvent aller à rebours des dynamiques historiques. La télévision, par exemple, se voit attribuer la capacité de retribaliser les individus et de les ramener à une culture de l'oralité. Cette ouverture permet d'analyser sur le registre de l'hybridation les interactions avec les médias et plus largement avec les dispositifs techniques contemporains. Mais penser l'hybridation médiatique nécessite de se départir de certains aspects de la pensée de McLuhan, notamment celle relative à la « température » des médias (Attallah, 1989, p. 295) :

«Par exemple, comment faut-il réagir devant la télévision «froide » qui est en train de devenir une télévision dite de haute définition. La télévision à haute définition de qualité visuelle comparable au cinéma exige moins de participation personnelle que l'ancienne télévision. Le média froid serait-il 
donc en train de devenir chaud par simple modification de ses caractéristiques technologiques ? [...] Lorsque l'électricité, média froid, est appliquée à l'imprimerie, média chaud, l'imprimerie refroidit-elle ou se réchauffe-t-elle?»

La grande popularité du «village global» de McLuhan est aujourd'hui amplement convoquée pour penser la globalisation à travers le maillage des télécommunications à l'échelle de la planète. Cette récupération mercantiliste et parfois futuriste va de pair avec les voies prometteuses dans lesquelles la recherche contemporaine s'engage en essayant de penser le medium, son contenu et l'environnement plus large dont seule la conjonction permet de comprendre l'interaction entre l'humain et le medium. Peut-être est-ce en conviant Harold Innis au secours de M. McLuhan que l'on peut espérer y parvenir.

Bibliographie

ATTALLAH P., 1989, « La théorie médiatique de Marshall McLuhan », Théories de la communication: Histoire, contexte, pouvoir, Québec, Presses de l'université du Québec, pp. 271-298.

BABE R., 2000, « The Communication Thought of Marshall McLuhan », Canadian Communication Thought: Ten Foundational Writers, Toronto, University of Toronto Press, pp. 266-306.

BELL D., 1973, The Coming of Post-Industrial Society, New York, Basic Books.

BOURDIEU P., 1980, Questions de sociologie, Paris, Éditions de Minuit.

BRZEZINSKI Z., 1970, Between Two Ages: America's Role in the Technetronic Era, New York, The Viking Press.

BUXTON W. J., 2004, « The "Values" Discussion Group at the University of Toronto, February-May 1949 », Canadian Journal of Communication, vol. 29, n 4, pp. 187-204.

BUXTON W. J., 2012, «The Rise of McLuhanism, The Loss of Innis-sense: Rethinking the Origins of the Toronto School of Communication ", Canadian Journal of Communication, vol. $37, \mathrm{n}^{\circ} 4$, pp.

577-593.

CAREY J. W., 1967, « Harold Adam Innis and Marshall McLuhan », The Antioch Review, vol. 27, n 1 , pp. 5-39.

CAREY J. et QUIRK J. J., 1970, « The Mythos of the Electronic Revolution. Part 1 », The American Scholar, vol. 39, n 1, pp. 219-241.

COUPLAND D., 2009, Marshall McLuhan, Montréal, Boréal.

GIEDION S., 1941, Space, Time and Architecture: The Growth of a New Tradition, Cambridge, Harvard University Press.

GIEDION S., 1948, Mechanization Takes Command: A Contribution to Anonymous History, New York, Oxford University Press.

HAVELOCK E., 1951, The Crucifixion of Intellectual Man, Boston, Beacon Press.

HAVELOCK E., 1963, Preface to Plato, Cambridge, Harvard University Press.

HAVELOCK E., 1981, Aux origines de la civilisation écrite en Occident, Paris, Maspero.

HEYER P., 1989, « Probing a Legacy: McLuhan's Communications/History 25 Years After », Canadian Journal of Communication, vol. $14, \mathrm{n}^{\circ} 4$, pp. 30-45.

INNIS H. A., 1950, Empire and Communications, Oxford, Clarendon Press. 
INNIS H. A., 1951, The Bias of Communication, Toronto, University of Toronto Press.

JEFFREY L., 1989, « The Heat and the Light: Towards a Reassessment of the Contribution of $\mathrm{H}$. Marshall McLuhan ", Canadian Journal of Communication, vol. 14, n, pp. 1-29.

KANE O., 2010, «Institution et légitimation d'une quasi-discipline : le triple destin (sciences, études et champ) de la communication ", Revue internationale de communication sociale et publique, vol. 16, n² 2, pp. 87-102, www.revuecsp.uqam.ca/numero/RICSP_3_4_2010.php

MACEY D., 2001, Dictionary of Critical Theory, Londres, Penguin Books.

MARCHAND P., 1989, « Some Comments on The McLuhan Papers in the National Archives of Canada », Canadian Journal of Communication, vol. 14, $n^{\circ} 4$, pp. 67-72.

MARCHAND P., 1990, Marshall McLuhan: The Medium and the Messenger, a Biography, Toronto, Vintage Books.

MARCHESSAULT J., 2005, Marshall McLuhan, Londres, Sage Publications.

McLUHAN E., 1989, « The New Science and the Old », Canadian Journal of Communication, vol. 14, $\mathrm{n}^{\circ}$ 4, pp. 80-91.

McLUHAN E., 2008, « Marshall McLuhan's Theory of Communication: The Yegg », Global Media Journal Canadian Edition, vol. 1, n 1, pp. 25-43.

McLUHAN M., 1943, The Classical Trivium: The Place of Thomas Nashe in the Learning of His Time, thèse de doctorat, Cambridge University.

McLUHAN M., 1946, « An Ancient Quarrel in Modern America », The Classical Journal, vol. 41, n 4, pp. 156-162.

McLUHAN M., 1951, The Mechanical Bride: Folklore of Industrial Man, Londres, Beacon Press.

McLUHAN M., 1967, The Medium is the Massage: An Inventory of Effects, New York, Bantam Books.

McLUHAN M., 1967, La Galaxie Gutenberg : La Genèse de l'homme typographique, Paris, Gallimard.

McLUHAN M., 1968, Pour comprendre les médias, Paris, Seuil.

McLUHAN M. et BARRINGTON N., 1972, Take Today: The Executive as Dropout, Don Mills, Longman.

McLUHAN M. et PARKER H., 1970, Counterblast, New York, Harcourt.

McNAMARA E., 1969, The Interior Landscape: The Literary Criticism of Marshall McLuhan, 1943-1962, New York et Toronto, McGraw-Hill.

MERZEAU L., 1998, « Ceci ne tuera pas cela », Les Cahiers de médiologie, vol. 2, n 6, pp. 27-39.

MEYROWITZ J., 1985, No Sense of Place, New York, Oxford University Press.

ONG W. J., 1970, Untitled Review of McLuhan's The Interior Landscape: The Literary Criticism of Marshall McLuhan 1943-1962, Criticism, n 12, pp. 244-251.

POSTMAN N., 1993, Technopoly: The Surrender of Technology to Culture, New York, Vintage Books. ROSENTHAL, R., 1969, McLuhan: Pro and Con, Baltimore, Penguin Books.

STAMPS J., 1995, Unthinking Modernity: Innis, McLuhan, and the Frankfurt School, Montréal, McGillQueen's University Press.

TOFFLER A., 1980, The Third Wave, Londres, Pan Books.

WILMOTT G., 1996, McLuhan, or Modernism in Reverse, Toronto, University of Toronto Press. 
WINTER J. P. et GLODMAN I., 1989, « Comparing the Early and Late Mcluhan to Innis's Political Discourse », Canadian Journal of Communication, vol. 14, $n^{\circ} 4$, pp. 92-100.

\section{NOTES}

1. Par souci de précision pour ce qui de la terminologie utilisée, nous utilisons media (sans « $\mathrm{s}$ » et non accentué) comme pluriel de medium (tout moyen de communication). Le terme « médias » est quant à lui réservé aux médias de masse (presse, radio, télévision, etc.).

2. Traduction de : «Will he continue to be viewed as a controversial figure, a marginal man from a marginal country who got it wrong, as current orthodoxy has it? Will he be assessed as an icon of the 1960's who contributed to a substantial shift in the ways ordinary people think about media? Or will his significance be seen to lie in his role as catalyst in the historical shaping of the modern "interdiscipline" of communications?»

3. Jeffrey (1989) considère que McLuhan est stéréotypé et utilisé comme un épouvantail au motif qu'il serait technodéterministe. Selon elle, on ne rend pas justice à la pensée du théoricien en le réduisant à ces deux clichés. Le travail de McLuhan dans The Gutenberg Galaxy s'inscrirait dans une tradition de recherche ancienne à laquelle il aurait apporté sa propre contribution et qui s'est intéressée de longue date aux conséquences du passage de l'oralité à l'écriture sur l'organisation culturelle, cognitive et sociale de l'humanité.

4. Selon Eric McLuhan, le fils de l'auteur canadien, le titre originel du livre était The Medium is the Message. Ce serait suite à une erreur typographique de l'imprimeur qui a mis un «a » à la place du «e» que le titre aurait été modifié ; M. McLuhan aurait ensuite suggéré un jeu de mots entre « message », « massage » et « mass age » en jouant sur l'idée de primat du medium.

5. Nous conservons ici le syntagme anglais pour éviter, en cas de traduction, la confusion avec la nouvelle critique française. Le new criticism est anglo-saxon et antérieur à la nouvelle critique qui a émergé en France au début des années 1960 suite aux travaux de Roland Barthes.

6. Traduction de : « the task of the critic is therefore to make "the closest possible examination of what the poem says as poem" ".

7. Traduction de : "The effects of new media on our sensory lives are similar to the effects of new poetry. They change not our thoughts but the structure of our world. »

8. McLuhan a, de manière récurrente, nié l'existence d'une théorie de la communication dans ses travaux, mais ce n'est pas l'avis de certains de ses spécialistes (McLuhan, 2008; Winter et Goldman, 1989).

9. Il s'agissait selon ses propres termes d'une tentative insensée de préserver l'héritage d'une culture livresque dans un âge dominé par l'électricité. De fait, une économie politique des médias n'a de sens pour lui que dans un cadre où prévalent des médias mécaniques (Babe, 2010), ce qui n'était manifestement plus le cas avec l'avènement de la télévision au moment où il développait ses écrits médiatiques.

10. Traduction de: "Somewhere in that 11-year span from 1951 to 1962, Marshall McLuhan became a technological determinist. »

11. Le chiasme serait un moyen de possible rédemption technologique pour le très catholique McLuhan. Pour des développements sur ce point et la perspective opposée d'Innis, voir Babe (2010, p. 299). 
12. Au Moyen Âge, le trivium était le corpus constitué de l'étude conjointe de la grammaire, de la rhétorique et de la logique. Le quadrivium regroupait de son côté l'étude des mathématiques, de la géométrie, de l'astronomie et de la musique.

13. L'écriture fait passer le sensorium humain du primat de l'auditif (oralité) à celui du visuel.

14. Traduction de : "A hot medium is one that extends a single sense in high definition; it is rich in specific information and leaves little to be filled by the audience. According to McLuhan print is hot, as is radio and film. In contrast, cool media are low definition in terms of information; they compel audience participation for resolution. This necessitates the involvement of more than one sense. Colloquial speech is cool, as well as the telephone and television. »

15. Comme l'écrit Coupland (2009, p. 294), « les médias transforment la société parce qu'ils transforment une faculté ou une capacité humaine universelle ».

16. Traduction de : «Every process pushed far enough tends to reverse or flip suddenly. »

17. Coupland (2009, p. 182) cite cet aphorisme de McLuhan qui sonne comme un blasphème : «L'utilisateur est le contenu. »

18. Traduction de : "The problem, of course, is that causality in history, if it exists at all, cannot be attributed to any single factor such as media, especially through the vast expanses of time dealt with in the Galaxy. »

19. Par ailleurs, quand McLuhan considère que ce n'est qu'avec le télégraphe que le message humain peut aller plus vite que le messager, il s'expose à la critique de Heyer (1989) qui lui rappelle que les signaux de fumée, le tam-tam et les relais vocaux ont rempli cette fonction bien avant la naissance du télégraphe électrique.

20. Traduction de : « interactive process, inherently connected to the growth of civilization, the emergence of universities, and the advent of new forms of public... Media, as discussed here, go well beyond the "economic staples", which McLuhan claimed to be the centrepiece of Innis' work in the history of communications."

21. Carey (1970) a été un passeur de premier plan pour la diffusion des travaux des deux théoriciens canadiens en raison de la clarté et de l'élégance de son style qui tranche avec le style abscons et hermétique de McLuhan et d'Innis.

22. L'hypothèse Sapir-Whorf, qui tire son nom des anthropologues Edward Sapir et Benjamin Lee Whorf, porte sur la relation entre langage et réalité. Elle illustre la thèse selon laquelle le langage n'est pas un simple instrument de description de la réalité ; c'est plutôt à travers elle que la réalité elle-même est construite et partagée.

23. Traduction de: "Nevertheless, any contemporary discussion of the role of television, including one based on, "heaven forbid", content, cannot help but address some aspect of McLuhan's perspective. Even complete avoidance of his position creates a situation whereby important considerations inevitably arise in the gap between the non-McLuhan view and the one it tries to sidestep or contradict. Despite its theoretical shortcomings Understanding Media is an important book. Its significance resides not in what it says about things, but in the things it brings together for the saying. "

24. Traduction de: "Institutionally, McLuhan contributed to the growth of interdisciplinary studies in a variety of ways. His interdisciplinary approach was evident in the diverse backgrounds of those invited to be part of the Seminars on Communications and Culture 1953-55 at the University of Toronto, funded by the Ford Foundation, and McLuhan's first publiclyrecognized achievement... The Ford Foundation grant in 1953 stimulated the collaboration and cross-fertilization known as The Toronto School of Communication. »

25. Traduction de: «The reason that I am admired in Paris and in some of the Latin countries is that my approach is rightly regarded as "structuralist". I have acquired that approach through Joyce and the Symbolists and used it in Mechanical Bride. Nobody except myself in the media field has ventured to use the structuralist or "existential" approach. It is a highbrow approach, and 
the schools of communication are uniformly hardware and flatfoot in their training and activities. »

26. De même que la théorie de l'information est la théorie communicationnelle des ingénieurs, la théorie de l'impact celle des psychologues et la théorie des interactions celle des sociologues (Attallah, 1989, p. 274).

27. L'exemple paradigmatique qu'il donne de l'ampoule électrique comme medium sans contenu est clair à cet égard. L'ampoule est sans contenu ni message mais, en tant que medium, elle réaménage complètement l'économie générale des échanges humains. Ce que l'ampoule éclaire et dans quel contexte devient moins pertinent à cet égard, l'essentiel résidant dans sa matérialité même.

\section{ABSTRACTS}

The hermeticism and aphoristic style of Marshall McLuhan probably affected the scientific reception of his work. Nevertheless, the writings of the Canadian author still continue to attract both criticisms and praise. In this paper, we provide a brief account of his contribution. After reviewing some of the ideas of thinkers to whom McLuhan himself is indebted, we address the posterity of his writings and evoke the most recurrent criticisms of his work. The paper ends by mentioning how McLuhan imported approaches mobilized in literary theory in the 1930s into media analysis and by calling on Harold Innis' approach to complement that of McLuhan.

Le sens aigu des aphorismes et l'hermétisme de Marshall McLuhan ont probablement nui à la réception scientifique de ses travaux. Néanmoins, les écrits de l'auteur canadien continuent de susciter critiques et dithyrambes. Dans cet article, nous effectuons à partir des sciences de la communication un bilan succinct de sa contribution. Après avoir passé en revue quelques-uns de ceux envers qui McLuhan lui-même est redevable, nous abordons la postérité de ses écrits et évoquons les critiques les plus récurrentes qui lui ont été adressées. L'article conclut en évoquant la manière dont McLuhan a importé de manière homologique dans l'analyse des media les approches mobilisées en théorie littéraire dans les années 1930 et en appelant en renfort l'approche de Harold Innis pour compléter celle de McLuhan.

Sobre la base de las ciencias de la comunicación, el presente artículo propone un sucinto balance de la contribución de Marshall McLuhan. Probablemente, el sentido agudo de los aforismos y del hermetismo de este autor ha afectado negativamente a la recepción de sus trabajos entre los científicos. También se puede establecer que los escritos del autor canadiense continúan suscitando críticas y laudatorios.Tras señalar algunos autores que influenciaron a McLuhan, se analizan diferentes textos y se evocan las críticas más recurrentes que le han sido destinadas. Finalmente, se concluye evocando la forma en que McLuhan ha importado, de manera homológica, las aproximaciones de teoría literaria en los años 1930, en el análisis de los medios. También se rescata la teoría de Harold Hinnis que completa la labor de McLuhan. 


\section{INDEX}

Mots-clés: McLuhan, théorie médiatique, medium, sensorium, déterminisme

Palabras claves: McLuhan, teoría de los medios, medio, sensorium, determinismo

Keywords: McLuhan, media theory, medium, sensorium, determinism

\section{AUTHOR}

\section{OUMAR KANE}

Oumar Kane est professeur au département de communication sociale et publique de l'université du Québec, à Montréal, et chercheur au Centre de recherche interuniversitaire sur la communication, l'information et la société (Cricis). Ses intérêts de recherche touchent notamment aux théories de la communication et à la philosophie de la technique. Il a récemment publié, en portugais, une analyse comparée des perspectives de Goody, Innis et McLuhan - « Escrita(s), mídias e tecnologias do intelecto: Notas sobre a atualidade dos trabalhos de Goody », Teorias dos Meios de Comunicação no Brasil e no Canadá, vol. 1, sous la dir. de Luiz Cláudio Martino et al., Salvador de Bahia, Edufba, 2014, pp. 53-72. 